\title{
Seven in every ten khat chewers in Gondar City had an intention to stop khat chewing: cross-sectional study using Transtheoretical Model
}

Asmamaw Adugna, Telake Azale and Simegnew Handebo* (1)

\begin{abstract}
Background: Khat chewing practice is rapidly expanding worldwide and currently, an estimated over 10 million people chew khat daily. The transtheoretical model explains how behavior change occurs across the stages of change. So, this study aimed at assessing the intention to stop khat chewing and associated factors among khat chewers in Gondar City, northwest Ethiopia.

Methods: Community-based cross-sectional study was conducted from March to April 2019 in Gondar City, northwest Ethiopia. Six hundred five khat chewers participated in the study from six randomly selected kebeles. The data were collected using structured and interviewer-administered questionnaire. Bivariate and multiple logistic regression models were fitted. Adjusted Odds ratio (AOR) with 95\% confidence interval (Cl) and $p$-values less than 0.05 were used to declare a significant association.

Results: Of the study participants, $69.3 \%$ (95\% Cl: 65.8-72.9) had the intention to stop khat chewing within 6 months or before. The majority of the participants (55.4\%) were at the contemplation stage. Higher self-reevaluation $[A O R=2.8,(95 \% \mathrm{Cl}: 1.6-5.0)]$, environmental reevaluation $[A O R=1.9,(95 \% \mathrm{Cl}: 1.1-3.3)]$ and social liberation $[A O R=1.8$, (95\% Cl: 1.0-3.1)] were associated with intention to quit khat chewing. In addition, using additional substances daily, khat dependency, and early age initiation of khat chewing were associated with lower intention to quit khat chewing.

Conclusion: The majority of the khat chewers had the intention to quit khat chewing. Increased self-reevaluation, environmental reevaluation, and social liberation process of changes were associated with enhanced intention of quitting khat chewing. Thus, stage-based interventions should be done to inspire khat chewers to realize their motivation of stopping khat chewing.
\end{abstract}

Keywords: Intention to quit, Khat, Trans theoretical model, Gondar, Ethiopia

\footnotetext{
* Correspondence: hsimegnew@yahoo.com

Department of Health Education and Behavioral Sciences, Institute of Public Health, College of Medicine and Health Sciences, University of Gondar, P.O. Box: 196, Gondar, Ethiopia
}

C C The Author(s). 2020 Open Access This article is licensed under a Creative Commons Attribution 4.0 International License, which permits use, sharing, adaptation, distribution and reproduction in any medium or format, as long as you give appropriate credit to the original author(s) and the source, provide a link to the Creative Commons licence, and indicate if changes were made. The images or other third party material in this article are included in the article's Creative Commons licence, unless indicated otherwise in a credit line to the material. If material is not included in the article's Creative Commons licence and your intended use is not permitted by statutory regulation or exceeds the permitted use, you will need to obtain permission directly from the copyright holder. To view a copy of this licence, visit http://creativecommons.org/licenses/by/4.0/. The Creative Commons Public Domain Dedication waiver (http://creativecommons.org/publicdomain/zero/1.0/) applies to the data made available in this article, unless otherwise stated in a credit line to the data. 


\section{Background}

Khat (Catha edulis) is an evergreen plant that grows at high altitudes between 1500 and $2000 \mathrm{~m}$ above the sea level. It is commonly grown throughout the year in the Horn of African and Middle East countries. Khat leaves have been used as a stimulant for recreational purposes for centuries in the Horn of Africa and the Arabian Peninsula [1-7]. It also used for social and religious purposes [8]. Khat contains psychoactive ingredients which affect consciousness, behavior, mood, and thinking processes of people [9]. These active ingredients have the potential to lead physical and psychological dependence [2, 8-10]. However, chewers believed that it will keep them alert, attain greater concentration, boosts pleasure, and enhances motivation [10].

Currently, khat chewing is becoming a common practice among young segment of the population in Ethiopia. A meta-analysis of 24 studies on the prevalence of khat chewing revealed that $23.2 \%$ of university students chew khat [11]. According to the Ethiopian Demographic and Health Survey (EDHS) 2016 report, 27\% of men and $12 \%$ of women ever chew khat and two in three of them chewed for 6 or more days in the last 30 days preceding the survey. The prevalence of khat chewing significantly varies among regions with the highest in Harari and lowest in Tigray, educational level and wealth status [12].

Chewing khat has multiple medical, sexual, economic and psychosocial problems. Studies revealed that habitual khat chewing results in cognitive impairment, learning problems, and behavioral abnormalities. It accounts for gastrointestinal tract problems like esophagitis, gastritis, and a delay in intestinal absorption. Moreover, khat chewing also causes dental, cardiovascular, and genitourinary problems [13-17]. Khat chewing has been found to affect the economy by decreasing the production as a result of tiredness and absenteeism. In some cases, workers go to lunch and engage in khat sessions, and may not return to work [18].

A qualitative study among former khat users identified numerous reasons that motivate khat chewers to make a decision on quitting khat use. The most common reasons were feeling of guilt for giving up prayers, feeling lost and neglecting family, accumulation of debts, work neglect and frequent absenteeism, and impaired health. Besides sexual and religious factors were the most pressing reasons for quitting [18]. The Transtheoretical Model (TTM) assumes that people do not change behaviors quickly and decisively; as well individuals who don't have a plan will remain stuck in the early stages of behavior change. The change in behavior, especially habitual behavior, occurs continuously through a cyclic process [19].

Studies on an unassisted quitting attempt among daily khat consumers showed that although khat chewers have the motivation and desire to quit, they encountered difficulties in maintaining abstinence. An unaided quitting attempt has low success and withdrawal symptoms are the major barriers to quit khat chewing. The discomfort following discontinuation of khat chewing increase the risk of relapse. Treatment and behavioral intervention are crucial to assist khat chewers' in quitting khat use [20, 21].

Empirical evidences suggest the TTM as the dominant model of health behavior change. The TTM enables to appreciate an individual's readiness to act on a new healthy behavior and describe how people move through the stages of behavioral changes. The four main constructs of the model are: stages of change, processes of change, decisional balance, and self-efficacy. The model indicates that individuals who are trying to change their behavior pass thru a series of five exclusive stages of change, namely: Pre-contemplation, Contemplation, Preparation, Action and Maintenance. The movement through these stages occurs in cyclic patterns. People apply cognitive, affective, and evaluative processes of change to progress through these stages of change [22]. Ten processes of change classified in two categories facilitate the transition from one stage to the next. The experiential processes of change are used primarily for the early stage transitions and include: consciousness raising, dramatic relief, environmental reevaluation, social liberation, and self-reevaluation. The behavioral processes of changes used in the later stage transitions are: stimulus control, helping relationships, counter conditioning, reinforcement management and self-liberation. Each process of change intervenes uniquely at one transition [22].

The current study focused on assessing the intention to stop khat chewing among chewers using TTM. Intention to quit khat chewing is conceptualized as a readiness to engage in the quitting process [23]. Although using intention to predict behavior was debatable [24], evidences support that intention to engage in the behavior enable to predict whether the behavior will occur or not [23]. Intention has low impact on behavior when control over the behavior is lacking, social reaction exist, and conditions are conducive to habit formation [25]. Planning, maintaining self-efficacy, and control of action serves to mediate between early intention and behavior. The higher the intention, the more likely the behavior will be performed [24, 26]. Theory-based measurement of behavior changes is important to organize thinking about the health problem as well as the development and refinement of interventions. Despite numerous studies on the prevalence of khat use were conducted in Ethiopia, quitting and intention to quit khat chewing area was not wellinvestigated. Therefore, this study assessed the magnitude of intention to quit khat chewing and associated factors among chat chewers in Gondar City, northwest Ethiopia. 


\section{Methods}

\section{Study setting and design}

A community-based cross-sectional study was conducted from March 15 to April 14, 2019, in Gondar, northwest Ethiopia. Gondar City is found in the central Gondar zone of Amhara regional state and located $727 \mathrm{~km}$ North West of Addis Ababa. There are 21 administrative kebeles with an estimated total population of 338,746 (160,522 males \& 178,223 females) [27]. The city is found $2133 \mathrm{~m}$ above mean sea level with annual rainfall up to 1200 cubic millimeters. In the city, there are 160 licensed and functional khat chewing houses and shops [28].

\section{Study population and sample size}

The study populations of this study were all khat chewers 18 and above years old and who lived in the city at least for 6 months. The sample size was determined by using the formula for the estimation of a single population proportion with an assumption of $95 \%$ confidence interval, $5 \%$ margin of error, and $50 \%$ of the expected proportion of khat chewer had an intention to quit khat chewing. To compensate for potential the non-response rate, $10 \%$ of the sample size was added. Then, the final sample size was 634. To recruit the study participants, first 6 kebeles were selected by a simple random sampling method. Then, all khat chewing houses and shops in the selected kebeles were identified and the sample size was proportionally allocated to each chewing houses and shops. Then, study participants were recruited randomly from each chewing house and shop until the sample size was met.

\section{Measures}

The first three of stages changes in TTM (pre-contemplation, contemplation, and preparation) are relevant to measure intention. Pre-contemplates were chewers who were not seriously thinking about quitting khat chewing in the next 6 months. Contemplates were the chewers who reported that they were thinking about quitting in the next 6 months but not in the next 30 days. Whereas, khat chewers at preparatory stages were those who plan to quit in the next 30 days and made an attempt in the last year [22]. So, intention to stop khat chewing is measured as dichotomous variable "If the participant had a plan to stop khat chewing within 6 months or before 'Yes'=1; Otherwise 'No' =0" i.e. contemplation and preparation stage of change.

In addition to socio-demographic variable and chewing history, five processes of change of khat chewing: consciousness-raising, dramatic relief, environmental reevaluation, social liberation, and self-reevaluation were assessed. Twenty Likert scale questions $(1=$ Strongly Disagree $2=$ Disagree $3=$ Neutral $4=$ Agree $5=$ Strongly Agree) were used to measure these processes of changes.
The mean score of the five dimensions was re-coded as low $(<3)$, medium (3) and high $(>3)$ [29, 30].

Self-efficacy, the confidence that individuals can manage temptations for not chewing khat, was measured by 20 Likert scale questions $(1=$ Not Very Confident $2=$ Not Confident $3=$ Neutral $4=$ Confident $5=$ Very Confident) and categorized as having low, intermediate and high selfefficacy if they had a mean score of $<3,3$ and $>3$ respectively. Decisional balance is the balance between the perceived advantages of chewing khat and the perceived disadvantages of chewing khat was measured by 18 Likert scale items (9 items for each) ( $1=$ Strongly Disagree $2=$ Disagree $3=$ Neutral $4=$ Agree $5=$ Strongly agree) and the total score was re-coded as positive, undecided and negative based on the result of total pros minus total cons score of chewing $[29,30]$.

Self-reported khat dependence, level of khat dependence, was measured by 10 Likert items questions ( $1=$ Strongly Disagree $2=$ Disagree $3=$ Neutral $4=$ Agree $5=$ Strongly agree) and the total score was recoded as low, medium and high dependence for a total score of $<3,3$ and $>3$ respectively $[29,30]$.

\section{Data collection procedures}

Interview administered, structured and translated questionnaire adapted from the different studies was used to collect the data [29-32]. The questionnaire was also pretested on $5 \%$ (32 participants) in Teda town. The reliability test for Cronbach's alpha of the consciousness raising (0.80), dramatic relief (0.78), environmental reevaluation (0.85), self-reevaluation (0.86), social liberation (0.74), self-efficacy (0.87), decisional balance (pros of khat chewing) (0.82), decisional balance (cons of khat chewing (0.85), and khat dependency scale (0.74) subscales were maintained. With the supervision of two supervisors and principal investigator, questionnaires were filled by 6 BSc degree holder Nurses. Intensive training was given to the data collectors and supervisors on the research tool, the purpose of the study, interviewing techniques and handling of ethical issues by the primary investigator.

\section{Data processing and analysis}

Data were entered into the EPI - info version 7 statistical software and exported to SPSS (Statistical Package for Social Science) version 20 for analysis. Descriptive statistics analysis was done to see the distribution of sociodemographic characteristics, process of changes, and intention to quit khat chewing. After the bivariable analysis was done, variables with $p$-values of $<0.2$ were entered into a multiple logistic regression model to identify predictors associated with intention to quit khat chewing. These factors were expressed by AOR (Adjusted Odds Ratio) with 95\% CI (Confidence Interval) and a 
probability value of type -1 error less of less than 0.05 was considered statistically significant.

\section{Results}

Socio demographic characteristics

Of the 634 selected participants, 605 were successfully interviewed, with a response rate of $95.4 \%$. Five hundred three $(83.1 \%)$ were males. The median age of the respondents was 27 years (23 and 34 years were the 1st and 3rd quartiles respectively). With regard to educational status, $270(44.6 \%)$ attended a diploma and above. The majority $384(63.5 \%)$ of them were single. Above one in each four (26.9\%) of the study participants had no job (See Table 1).

\section{Khat dependency and chewing history}

Of the total respondents, 263(43.5\%) had high khat dependence and 310(51.2\%) of the total respondents had a low dependency. The mean age for initiation of Khat chewing was $20.42(\mathrm{SD} \pm 4.3)$ years. The mean duration of Khat chewing was 8.67 ( $\mathrm{SD} \pm 6.5$ ) years. Two hundred thirty (38.0\%) of the participants chewed khat for 4.39 $(\mathrm{SD} \pm 2.6)$ hours per week. With regard to the purpose of chewing most of the participants, 317 (52.4\%) chewed khat for recreation and $129(21.3 \%)$ for reading. For about three-fourth 447 (73.9\%) of the participants, khat chewing was a means of social interaction. Chewing was combined with cigarette smoking for 220 (36.4\%) and alcohol drinking for 170 (28.1\%) participants.

\section{Stages of change}

Out of the total participants, 419 (69.3\%) (95\% CI: 65.872.9) have an intention to stop khat chewing at least within the next 6 months. The majority of the study participants $335(55.4 \%)$ were on the contemplation stage. Whereas $84(13.9 \%)$ were on the preparation stage and the rest $186(30.7 \%)$ were on the pre-contemplation stage.

\section{Processes of change and self-efficacy}

Nearly half of the participants had low consciousnessraising (48.3\%) and high dramatic relief (46.8\%) scores. The majority of them had high self-reevaluation (60.3\%) social liberation (53.4\%) and environmental reevaluation (62.5\%) scores. The respondent's self-efficacy to stop khat chewing revealed that half $(50.9 \%)$ had low selfefficacy and 276 (45.6\%) had high self-efficacy. Only $21(3.5 \%)$ of them had medium self-efficacy to stop khat chewing (See Table 2).

\section{Decisional balance to stop khat chewing}

This study found that for the majority $(57.4 \%)$ of the respondent, the mean score of cons of khat chewing was higher than the pros score (negative decisional balance).
Table 1 Socio demographic characteristics of Khat chewers in Gondar city, north west Ethiopia; 2019 ( $n=605)$

\begin{tabular}{|c|c|c|}
\hline Socio demographic characteristics & Frequency & Percent \\
\hline \multicolumn{3}{|l|}{ Sex } \\
\hline Male & 503 & 83.1 \\
\hline Female & 102 & 16.9 \\
\hline \multicolumn{3}{|l|}{ Age in years } \\
\hline $18-24$ & 200 & 33.1 \\
\hline $25-29$ & 159 & 26.3 \\
\hline $30-34$ & 106 & 17.5 \\
\hline 35 and above & 140 & 23.5 \\
\hline \multicolumn{3}{|l|}{ Religion } \\
\hline Christian & 377 & 62.3 \\
\hline Muslim & 191 & 31.6 \\
\hline Protestant & 22 & 3.6 \\
\hline Other (Catholic, no religion) & 15 & 2.5 \\
\hline \multicolumn{3}{|l|}{ Ethnicity } \\
\hline Amhara & 513 & 84.8 \\
\hline Tigrie & 38 & 6.3 \\
\hline Oromo & 37 & 6.1 \\
\hline Other (Kimant, Gurage, Sidama) & 17 & 2.8 \\
\hline \multicolumn{3}{|l|}{ Educational status } \\
\hline Can't read \& write & 30 & 5.0 \\
\hline Read \& write & 28 & 4.6 \\
\hline Primary education & 55 & 9.1 \\
\hline Secondary education & 222 & 36.7 \\
\hline Diploma \& above & 270 & 44.6 \\
\hline \multicolumn{3}{|l|}{ Occupation } \\
\hline Government employee & 89 & 14.7 \\
\hline Merchant & 132 & 21.8 \\
\hline Self-employee & 93 & 15.4 \\
\hline Student & 85 & 14.0 \\
\hline Job seeker & 163 & 26.9 \\
\hline Other (driver, Guard) & 43 & 7.1 \\
\hline \multicolumn{3}{|l|}{ Marital status } \\
\hline Single & 384 & 63.5 \\
\hline Married & 174 & 28.8 \\
\hline Divorced & 21 & 3.5 \\
\hline Other (separated, widowed) & 26 & 4.1 \\
\hline \multicolumn{3}{|l|}{ Monthly income in birr } \\
\hline$<2000$ & 302 & 49.9 \\
\hline $2000-3500$ & 134 & 22.1 \\
\hline$>3500$ & 169 & 28.0 \\
\hline
\end{tabular}


Table 2 Process of changes and self-efficacy of stopping khat chewing among khat chewers in Gondar city, northwest Ethiopia; $2019(n=605)$

\begin{tabular}{|c|c|c|}
\hline Variable & Frequency & Percent (\%) \\
\hline \multicolumn{3}{|c|}{ Consciousness raising } \\
\hline Low & 292 & 48.3 \\
\hline Medium & 53 & 8.8 \\
\hline High & 260 & 43.0 \\
\hline \multicolumn{3}{|c|}{ Dramatic relief } \\
\hline Low & 273 & 45.1 \\
\hline Medium & 49 & 8.1 \\
\hline High & 283 & 46.8 \\
\hline \multicolumn{3}{|c|}{ Self-reevaluation } \\
\hline Low & 206 & 34.0 \\
\hline Medium & 34 & 5.6 \\
\hline High & 365 & 60.3 \\
\hline \multicolumn{3}{|c|}{ Social liberation } \\
\hline Low & 215 & 35.5 \\
\hline Medium & 67 & 11.1 \\
\hline High & 323 & 53.4 \\
\hline \multicolumn{3}{|c|}{ Environmental reevaluation } \\
\hline Low & 190 & 31.4 \\
\hline Medium & 37 & 6.1 \\
\hline High & 378 & 62.5 \\
\hline \multicolumn{3}{|l|}{ Self-efficacy } \\
\hline Low & 308 & 50.9 \\
\hline Medium & 21 & 3.5 \\
\hline High & 276 & 45.6 \\
\hline
\end{tabular}

On the other hand, for $38.3 \%$ of them, the mean score of pros of khat chewing was higher than the cons (positive decisional balance) and 26 (4.3\%) of respondents were undecided.

\section{Factors associated with intention to stop khat chewing}

As indicated in Table 3, in multiple logistic regression analysis age at initiation of khat chewing, frequency of additional drugs use, environmental reevaluation, selfreevaluation, and social liberation variables were significantly associated with intention to quit khat chewing. The odds of intention to quit khat chewing increased among adults who started chewing at an older age compared to those started chewing in early age. Adults who started chewing khat in the age group 16-20 years $[\mathrm{AOR}=3.2$, $(95 \% \mathrm{CI} ; 1.5-6.9)], 21-25$ years $[\mathrm{AOR}=2.8$, (95\% CI; 1.1-6.9)], and older than 26 years $[\mathrm{AOR}=4.8$, (95\% CI; 1.5-15.5)] had higher odds of intention to quit khat chewing compared to those who started chewing at age 15 and below. The odds of intention to quit khat chewing among adults who use additional substances (cigarette, hashish, alcohol) daily was $63 \%$ less likely than those who use sometimes $[\mathrm{AOR}=0.37$, (95\% CI; 0.16$0.85)]$. Adults who had high environmental reevaluation score were about 2 times more likely have the intention to quit chewing than those who had low environmental reevaluation score $[\mathrm{AOR}=1.9,(95 \% \mathrm{CI}: 1.1-3.3)]$. Khat chewers who had high self-reevaluation score were 3 times more likely have the intention to quit chewing than those who had low self-reevaluation score $[\mathrm{AOR}=$ 2.8 (95\% CI: 1.6-5.0)]. Participants who had high social liberation score were about 2 times more likely have the intention to stop khat chewing than those with low social liberation score $[\mathrm{AOR}=1.8,(95 \% \mathrm{CI}: 1.0-3.1)]$. Respondents who had a high khat dependence were 39\% less likely to have the intention to stop khat chewing as compared to those low khat dependence $[\mathrm{AOR}=0.61$, (95\%CI: 0.38-0.99)] (See Table 3).

\section{Discussion}

Despite finding on the prevalence of khat chewing, study reports on intention to stop chewing and quitting attempts aid policymakers and programmers in designing appropriate interventions at different levels. In this study majority of participants $(69.3 \%)$ had an intention to stop khat chewing within the next 6 months or before. This finding is consistent with a study conducted in Dessie city (Ethiopia) [29]. On the other hand, this finding is higher than studies done in Saudi Arabia (29.5\%) [33], male Yemeni adult residents in the UK (46\%) [34] and UK (55\%) [35]. This could be due to differences in sociodemographic characteristics of the study population. The other reason might be due to difference in the way intention to quit khat chewing was measured. Intention to quit khat chewing was measured by a single question in Saudi Arabia, Yemeni, and UK studies. While in our study intention was measured by using the staging algorithm developed by Prochaska, Diclemente, and colleagues (contemplation and preparation stages) [19].

In the present study, the mean age of initiation of khat chewing was $20.42(\mathrm{SD} \pm 4.3)$ years. This finding is in line with previous studies conducted in Dessie (Ethiopia) [29], Saudi Arabia [33], and the UK [35] but higher than the finding reported among Yemeni adults in the UK [34]. In this study, khat chewers who started chewing at older age had increased odds of intention to quit khat chewing than those who start at a younger age. Adults who started chewing at an earlier age had a prolonged khat chewing experience and higher khat dependence level. As a result, khat chewers who started chewing at a younger age (15 years and below) less likely intend to stop chewing. Likewise, this study also found that intention to stop khat chewing was lower among chewers who had high khat dependence levels. A study done in Saudi Arabia reported that low intention to stop 
Table 3 Multiple logistic regression analysis of factors associated with intention to stop khat chewing among Khat chewers in Gondar city, northwest Ethiopia; 2019

\begin{tabular}{|c|c|c|c|c|c|}
\hline \multirow[t]{2}{*}{ Variable } & \multirow[t]{2}{*}{ Category } & \multicolumn{2}{|c|}{ Intention to stop khat chewing } & \multirow{2}{*}{$\begin{array}{l}\text { COR, } \\
95 \% \mathrm{Cl}\end{array}$} & \multirow[t]{2}{*}{ AOR, $95 \% \mathrm{Cl}$} \\
\hline & & Yes & No & & \\
\hline \multirow[t]{4}{*}{ Age at start of khat chewing } & $\leq 15$ & 22 & 32 & 1 & 1 \\
\hline & $16-20$ & 243 & 107 & $2.3(1.8-2.9)$ & $3.2(1.5-6.9) *$ \\
\hline & $21-25$ & 96 & 34 & $2.8(1.9-4.2)$ & $2.8(1.1-6.9) *$ \\
\hline & $\geq 26$ & 58 & 13 & $4.4(2.4-8.1)$ & $4.8(1.5-15.5) *$ \\
\hline \multirow[t]{4}{*}{ Frequency of additional drug use } & Sometimes & 96 & 30 & 1 & 1 \\
\hline & $1-3$ days & 67 & 24 & $2.8(1.8-4.5)$ & $0.51(0.2-1.2)$ \\
\hline & 4-6 days & 76 & 30 & $2.5(1.7-3.9)$ & $0.63(0.26-1.5)$ \\
\hline & Day to day & 84 & 53 & $1.6(1.1-2.2)$ & $0.37(0.16-0.8) *$ \\
\hline \multirow[t]{3}{*}{ Khat dependency } & Low & 222 & 88 & 1 & 1 \\
\hline & Medium & 20 & 12 & $1.7(0.8-3.4)$ & $0.72(0.25-2.0)$ \\
\hline & High & 177 & 86 & $2.5(2.0-2.7)$ & $0.61(0.38-.9) *$ \\
\hline \multirow[t]{3}{*}{ Environmental Reevaluation } & Low & 87 & 101 & 1 & 1 \\
\hline & Medium & 28 & 10 & $2.7(1.3-5.6)$ & $2.0(.78-5.4)$ \\
\hline & High & 302 & 77 & $4.0(3.1-5.2)$ & $1.9(1.1-3.3) *$ \\
\hline \multirow[t]{3}{*}{ Self-Reevaluation } & Low & 94 & 111 & 1 & 1 \\
\hline & Medium & 25 & 12 & $2.4(1.1-5.0)$ & $1.6(0.6-4.2)$ \\
\hline & High & 298 & 65 & $4.6(3.5-6.0)$ & $2.8(1.6-5.0) *$ \\
\hline \multirow[t]{3}{*}{ Social Liberation } & Low & 110 & 104 & 1 & 1 \\
\hline & Medium & 42 & 27 & $1.6(1.0-2.6)$ & $1.1(.53-2.3)$ \\
\hline & High & 265 & 57 & $4.7(3.5-6.2)$ & $1.8(1.01-3.1) *$ \\
\hline
\end{tabular}

*Statistically Significant at $p$-value $<0.05$

khat chewing is potentially due to high level of khat dependence. This may indicate the gaps in prevention strategies on substance abuse in general and khat use in particular [33].

The present study found that individuals who use additional drugs daily (cigarette, hashish, alcohol) were less likely intend to quit khat chewing as compared to those who use sometimes. Since using one would tempt the use of others concurrent substance use remains as a main barrier of intention to quit and quitting process. In line with this, the systematic review and meta-analysis among university students in Ethiopian reported alcohol drinking and cigarette smoking as a predictor of khat chewing [11]. This finding was consistent with a qualitative study done among khat users and healthcare professionals in London [36]. In contrast, other drug use was not associated with khat use or quitting in the study done among khat quitters in the Jazan area of Saudi Arabia [18].

In TTM the processes of change are the covert and overt activities that people use to progress through stages of changes [22]. The current study revealed that the participant who had high environmental reevaluation, self-reevaluation and social liberation more likely intend to quit khat chewing than their counterparts. Environmental reevaluation is realizing the negative impact of khat chewing on one's own social and/or physical environment $[37,38]$. In our study, adults who had high environmental reevaluation scores had two times more likely have the intention to quit khat chewing compared to those who had a low environmental reevaluation score. Similarly, individuals who had higher social liberation score were two times more likely have the intention to stop khat chewing than those with low social liberation score. Self-liberation is realizing that social norms are changing in the direction of supporting health behavior change [37, 38]. This finding is inconsistent with studies done in Dessie city, which reported medium environmental reevaluation and social liberation score are associated with increased intention to quit khat chewing [29]. Intention to quit does not vary between high, medium and low environmental reevaluation and social liberation process of changes in a study done in Dire Dawa town [30]. The possible reason might be the difference in sociocultural and demographic factors between the study areas.

On the other hand, individuals with high selfreevaluation were 3 times more likely to have the 
intention to quit khat chewing than those with low selfreevaluation. Self-reevaluation is realizing that behavior change is an important part of one's identity [37, 38]. This result is in line with the a study done in Dire Dawa town [30] and whereas, contradict with finding in Dessie town [29]. The observed difference might be due to sociocultural variation between the study populations. Other variables in TTM like, consciousness-raising, dramatic relief, self-efficacy, and decisional balance were not significantly associated with intention to quit khat chewing.

\section{Limitation of the study}

There are a few limitations to this study. First, in the TTM the lines between the stages are both stable and open to change. From the stage algorithm of the model precontemplation, contemplation and preparation stages were included in this study and 6 months of period used as criteria for determine intention. Second, since khat chewing behavior is sensitive there may be social desirability that overestimates the intention to quit chewing. We have provided full anonymity to all participants. As such, we believe that the response biases have been kept to a minimum. Being a cross-sectional study may not allow establishing to draw causal relationships among the variables.

\section{Conclusion}

The majority of the khat chewers had intention to stop khat chewing at least within the next 6 months. Age of khat chewing initiation, daily use of additional drugs, khat dependency, environmental reevaluation, selfreevaluation, and social liberation were significant predictors of intention to stop khat chewing. Thus, stagebased interventions should be taken to personalized risk, alter risk perception, clarify values and misconceptions. In addition to intervention to decrease substance abuse, skill training and modeling (who have previously overcome difficult barriers) need to be emphasized to enhance khat chewers' intention to stop chewing. On the other hand, it is important to assist chewers' in their quit attempts to increase success rates. Reducing khat chewing habit have an important effect on health and economic development.

\section{Acknowledgements}

We would like to thank the study participants for their willingness to participate in the study. Our appreciation will also go to the data collectors.

\section{Authors' contributions}

AA conceived the study, coordinated data collection, and carried out the statistical analysis and drafted the manuscript. TA SH participated in the drafting research, statistical analysis, and review the drafted the manuscript. All authors read and approved the final manuscript.

\section{Funding}

The authors received no specific funding for this work.

\section{Availability of data and materials}

The datasets used in the current study are available from the corresponding author on reasonable request.

\section{Ethics approval and consent to participate}

This work has been approved by the ethical review Board of University of Gondar, Collage of Medicine and Health Science, Institute of Public Health. Protocol number: 180/2019. A formal letter of Permission was obtained from Gondar town health department and submitted to the owners of khat chewing houses and shops. Verbal informed consent was obtained from all chewing houses and shops. After explaining the purpose of the study oral informed consent was obtained from the study participants. The

participation was voluntary, and personal identifiers were not included in the questionnaires. Oral consent was approved by the Institutional Review Board. The confidentiality and anonymity of participant's information kept and the data were stored in a password locked computer. Health information was given for participant about the effect of khat by data collectors.

\section{Consent for publication}

Not applicable.

\section{Competing interests}

The authors declare that they have no conflict of interest.

Received: 18 May 2020 Accepted: 23 November 2020

Published online: 02 December 2020

\section{References}

1. National Drug Intelligence Center: Khat (Catha edulis): Intelligence Bulletin 2003 [http://www.justice.gov/ndic/pubs3/3920/index.htm]. No. 2003. L0424002.

2. Kalix P. Cathinone, a natural amphetamine. Pharmacol Toxicol. 1992;70:77-86.

3. Al-Mugahed L. Khat chewing in Yemen: turning over a new leaf. Bull World Health Organ. 2008:741-2.

4. Brooke C. Khat (Catha edulis): its production and trade in the Middle East. Geogr J. 1960;126(1):52-9.

5. Corkery JM, Schifano F, Oyefeso A, Ghodse AH, Tonia T, Naidoo V, Button J. Overview of literature and information on" khat-related" mortality: a call for recognition of the issue and further research. Ann Ist Super Sanita. 2011;47: 445-64.

6. Manghi RA, Broers B, Khan R, Benguettat D, Khazaal Y, Zullino DF. Khat use: lifestyle or addiction? J Psychoactive Drugs 2009:41(1):1-0,

7. Anderson D, Carrier NC. Khat: social harms and legislation: a literature review. Home Office; 2011

8. Hussain MAA. Health and Soci-economic Hazard associated with Khat consumption. J Community Med. 2013;15(1):3-11.

9. Wabe NT. Chemistry, pharmacology, and toxicology of khat (catha edulis forsk): a review. Addict Health. 2011:3(3-4):137.

10. WHO (2004) Neuroscience of psychoactive substances and dependency.

11. Gebrie A, Alebel A, Zegeye A, Tesfaye B. Prevalence and predictors of khat chewing among Ethiopian university students: a systematic review and meta-analysis. PLoS One. 2018 Apr 12;13(4):e0195718.

12. Central Statistical Agency (CSA) [Ethiopia] and ICF. Ethiopia Demographic and Health Survey 2016. Maryland: CSA and ICF; 2016.

13. Colzato LS, Ruiz MJ, van den Wildenberg WP, Hommel B. Khat use is associated with impaired working memory and cognitive flexibility. PLoS One. 2011;6(6):e20602.

14. al'Absi M, Khalil NS, Al Habori M, Hoffman R, Fujiwara K, Wittmers L. Effects of chronic khat use on cardiovascular, adrenocortical, and psychological responses to stress in men and women. Am J Addict. 2013;22(2):99-107.

15. Al-Habori M. The potential adverse effects of habitual use of Catha edulis (khat). Expert Opin Drug Saf. 2005;4(6):1145-54.

16. Al-Motarreb A, Briancon S, Al-Jaber N, Al-Adhi B, Al-Jailani F, Salek MS, Broadley KJ. Khat chewing is a risk factor for acute myocardial infarction: a case-control study. Br J Clin Pharmacol. 2005;59(5):574-81.

17. Ageely HM. Health and socio-economic hazards associated with khat consumption. J Fam Community Med. 2008;15(1):3.

18. Alsanusy R, El-Setouhy M. Why would khat chewers quit? An in-depth, qualitative study on Saudi Khat quitters. Subst Abus. 2013;34(4):389-95.

19. Wayne W. LaMorte, The Transtheoretical Model (Stages of Change); Boston University School of Public Health, 2019. http://sphweb.bumc.bu.edu/otlt/ 
MPH-Modules/SB/BehavioralChangeTheories/BehavioralChangeTheories6. html Accessed 17 Jan 2020.

20. Duresso SW, Bruno R, Matthews AJ, Ferguson SG. Khat withdrawal symptoms among chronic khat users following a quit attempt: an ecological momentary assessment study. Psychol Addict Behav. 2018;32(3): 320-6.

21. Duresso SW, Bruno R, Matthews AJ, Ferguson SG. Stopping khat use: predictors of success in an unaided quit attempt. Drug Alcohol Rev. 2018; 37:S235-9.

22. Prochaska JO, Velicer WF. The transtheoretical model of health behavior change. Am J Health Promot. 1997;12(1):38-48.

23. Fishbein M. A reasoned action approach to health promotion. Med Decis Mak. 2008;28(6):834-44.

24. Manski CF. The use of intentions data to predict behavior: a best-case analysis. J Am Stat Assoc. 1990;85(412):934-40.

25. Webb TL, Sheeran P. Does changing behavioral intentions engender behavior change? A meta-analysis of the experimental evidence. Psychol Bull. 2006;132(2):249.

26. Sniehotta FF, Scholz U, Schwarzer R. Bridging the intention-behaviour gap: planning, self-efficacy, and action control in the adoption and maintenance of physical exercise. Psychol Health. 2005;20(2):143-60.

27. Gondar City Health Office. The 2017/2018 Annual Report. (Unpublished document).

28. Gondar City Trade, Industry, and Market Development Office. The 2017/ 2018 Annual Report. (Unpublished document).

29. Estifanos M, Azale T, Slassie MG, Amogne G, Kefale B. Intention to stop khat chewing and associated factors among khat chewers in Dessie city, North Eastern Ethiopia. Epidemiology (Sunnyvale). 2016;6(250):2161-1165.

30. Girma E, Assefa T, Deribew A. Cigarette smokers' intention to quit smoking in Dire Dawa town Ethiopia: an assessment using the Trans theoretical Model. BMC Public Health. 2010;10(1):320

31. West R. Finding better ways of motivating and assisting smokers to stop: research at the CRUK health behaviour research Centre. Eur Health Psychol. 2008;10:54-8.

32. Velicer WF, DiClemente CC, Prochaska JO, Brandenburg N. Decisional balance measure for assessing and predicting smoking status. J Pers Soc Psychol. 1985;48(5):1279.

33. Abdelwahab SI, Alsanosy RM, Rahim BE, Mohan S, Taha S, Mohamed Elhassan M, El-Setouhy M. Khat (Catha edulis Forsk.) dependence potential and pattern of use in Saudi Arabia. BioMed Res Int. 2015;2015:604526.

34. Kassim S, Islam S, Croucher R. Validity and reliability of a severity of dependence scale for khat (SDS-khat). J Ethnopharmacol. 2010;132(3):570-7.

35. Kassim S, Rogers N, Leach K. The likelihood of khat chewing serving as a neglected and reverse 'gateway'to tobacco use among UK adult male khat chewers: a cross sectional study. BMC Public Health. 2014;14(1):448.

36. Begum S, Bogosian A, McBain H. The experiences of people who quit khat and the health care professionals who support them. Addict Health. 2019; 11(4):243-55.

37. Prochaska JO, Goldstein MG. Process of smoking cessation: implications for clinicians. Clin Chest Med. 1991;12(4):727-35.

38. DiClemente C, Delahanty J, Jean F, Earley M, Garay M, Preston G, Meredith H, Angela P, Dan R, Kristina S, Onna V, Katherine W: Health and addictive behaviors: investigating transtheoretical solutions, Transtheoretical model of behavior change measures. 1994 [http://www.umbc.edu/psyc//habits/ content/ttm_measures/index.html]. Accessed on 30 Sept 2008.

\section{Publisher's Note}

Springer Nature remains neutral with regard to jurisdictional claims in published maps and institutional affiliations.

Ready to submit your research? Choose BMC and benefit from:
- fast, convenient online submission
- thorough peer review by experienced researchers in your field
- rapid publication on acceptance
- support for research data, including large and complex data types
- gold Open Access which fosters wider collaboration and increased citations
- maximum visibility for your research: over 100M website views per year
At BMC, research is always in progress.
Learn more biomedcentral.com/submissions

